\title{
Which type of scaling system is best?
}

\author{
Abstracted from \\ Slot DE, Koster TJ, Paraskevas S, Van der Weijden GA. \\ The effect of the Vector scaler system on human teeth: a systematic review. \\ Int J Dent Hyg 2008; 6: 154-165 \\ Address for correspondence: DE Slot, Academic Centre for Dentistry Amsterdam, \\ Department of Periodontology, Louwesweg 1, 1066 EA Amsterdam, \\ The Netherlands. E-mail: d.slot@acta.nl
}

\section{Question: How effective is the Vector ultrasonic scaler on human teeth in vitro and in vivo compared with conventional ultrasonic instruments and/ or hand instrumentation?}

Data Sources Medline and the Cochrane Central register of Controlled Trials were searched for relevant studies.

Study selection Randomised controlled trials and controlled clinical trials conducted in vitro or in vivo on human teeth, which used plaque, bleeding, gingivitis or pocket depth, clinical attachment level, microbiological findings, patient perception and treatment time as outcome measures, were included. Case reports, letters and narrative or historical reviews were excluded and only English-language papers were considered.

Data extraction and synthesis The studies included differed in design and outcome so a qualitative summary was presented.

Results Fifteen studies met the inclusion criteria. The Vector (Dürr Dental, Bietigheim-Bissingen, Germany) ultrasonic scaler (VUS) provided clinical and microbiological periodontal healing results comparable to scaling and root planing and a conventional ultrasonic system in moderately deep pockets.

Conclusions The VUS may be used as a gentle root debridement device for supportive periodontal therapy, as an alternative to other conventional ultrasonic systems. The operator should consider, however, the extra time needed for instrumentation.

\section{Commentary}

All new technology requires validation against existing methods, a few years usually being necessary to gather sufficient independent evidence. The authors of this review assess the effectiveness of the VUS compared with hand or powered instrumentation in the treatment of periodontal disease. The first paper describing the VUS appeared following a study of 50 patients in 2003, and there are now over a dozen papers comparing this system with hand or ultrasonic instruments. Often the main question of reviews can be too narrow, resulting in the omission of relevant research but, here, the authors ask a broad question and can still only include 15 papers. There is so much heterogeneity between studies that the authors are also restricted to making broad comments.

Scaling and root planning should be the initial treatment of periodontal disease, ${ }^{1}$ but particular areas can be difficult to debride, such as deeper pockets ${ }^{2}$ and furcations. ${ }^{3}$ Ultrasonic instruments may be better in these areas.

This paper correctly surmises that the VUS may be just as good as conventional instrumentation, leaving us with the question of cost vs benefit, ease of use, time required and patient acceptance. The first studies suggested that the effectiveness depended on the inserts used and irrigation fluid, as seen with conventional ultrasonics. Kasaj et al. ${ }^{4}$ noted that treatment took four times as long if the VUS was used, although other studies suggested times were fairly similar. There is a further suggestion that the VUS may be "gentler" and remove much less of the root surface. There remain, however, a number of limitations that do not help support its purchase.

Practice points

- VUS can be as effective as hand or ultrasonic debridement

- Treatment may take longer, be less effective and depend on the correct choice of insert and irrigation fluid, so the VUS may ultimately not be as useful as the more common instruments.

Ivan Darby

Melbourne Dental School, University of Melbourne, Parkville, Victoria, Australia

1. Badersten A, Nilveus R, Egelberg J. Effect of non-surgical periodontal therapy. II. Severely advanced periodontitis. / Clin Periodontol 1984; 11: 63-76.

2. Waerhaug $\mathrm{J}$. Healing of the dento-epithelial junction following subgingival plaque control. II. As observed on extracted teeth. J Periodontol 1978; 49: 119-134.

3. Loos B, Claffey N, Egelberg J. Clinical and microbiological effects of root debridement in periodontal furcation pockets. / Clin Periodontol 1988; 15: 453-463.

4. Kasaj A, Chiriachide A, Willershausen B. The adjunctive use of a controlled-release chlorhexidine chip following treatment with a new ultrasonic device in supportive periodontal therapy: a prospective, controlled clinical study. Int J Dent Hyg 2007; 5: $225-231$.

Evidence-Based Dentistry (2009) 10, 45. doi:10.1038/sj.ebd.6400648 